\title{
Civilisations
}

Revue internationale d'anthropologie et de sciences

humaines

$65 \mid 2016$

Figures du malentendu

\section{Des malentendus à (bien) interpréter}

\section{Maria Luisa Ciminelli}

\section{OpenEdition \\ Journals}

Édition électronique

URL : http://journals.openedition.org/civilisations/4104

DOI : $10.4000 /$ civilisations.4104

ISSN : 2032-0442

\section{Éditeur}

Institut de sociologie de l'Université Libre de Bruxelles

\section{Édition imprimée}

Date de publication : 19 décembre 2016

Pagination : 81-106

ISBN : 2-9602017-0-3

ISSN : 0009-8140

\section{Référence électronique}

Maria Luisa Ciminelli, « Des malentendus à (bien) interpréter », Civilisations [En ligne], 65 | 2016, mis en ligne le 19 décembre 2019, consulté le 15 janvier 2021. URL : http://journals.openedition.org/ civilisations/4104; DOI : https://doi.org/10.4000/civilisations.4104 


\title{
Des malentendus à (bien) interpréter*
}

\author{
Maria Luisa CIMINELLI
}

Résumé: La traduction, considérée par plusieurs auteurs comme paradigmatique pourl'entreprise anthropologique, est aussi une pratique habituelle sur le "terrain ", où les malentendus, et ce qui peut être interprété comme une manipulation volontaire, peuvent ensuite s'avérer des clés pour la vérification des résultats de la recherche et, plus généralement, des instruments de compréhension. C'est dans cette perspective que l'auteure réexamine la transcription d'un entretien s'étant déroulé en 1992, au début de sa recherche dans le Bélédougou (Mali), en mettant en contraste le dialogue en français entre elle et l'interprète bambara avec sa traduction française du dialogue, en langue bamanan, entre l'interprète et la guérisseuse interviewée, une jinètigi (prêtresse du culte des jinè).

Mots-clés : traduction, Bamanan, interprétation, pragmatique, malentendu.

Abstract: Often regarded as paradigmatic of anthropological knowledge, translation is an ordinary practice in fieldwork, where different types of misunderstanding and even what could be interpreted as voluntary manipulation may, however, prove to be precious insights into understanding. In that light, the author re-examines the transcription of a conversation held in 1992 in Bamana and French languages, contrasting her translation of Bamana sentences with the translation formulated by the interpreter.

Keywords: translation, Bamana, interpretation, pragmatics, misunderstanding.

* Je voudrais ici exprimer des remerciements pour la lecture attentive, les précieuses et remarquables suggestions qui m'ont été consacrées, soit par les éditeurs de ce numéro et de la revue, soit par les referees anonymes. Nombreuses sont les suggestions qui auraient mérité d'être développées dans cette publication ; ce qui ne fut malheureusement possible pour des raisons de temps, d'espace et de bien d'autres natures. Je me réserve toutefois l'engagement de les reprendre dans de prochaines publications ou écritures. Je souhaite également adresser toute ma gratitude au Prof. Samou Camara pour sa contribution tant dans la transcription que dans la traduction du texte bamanankan, dans un soutien amical et continu. 
C'était en 1990. Lorsque, pour la première fois, je me rendis dans le Bélédougou ${ }^{1}$, parcourant en jeep la route, pas encore goudronnée à l'époque, qui relie Bamako à Kolokani, je demandai au chauffeur pourquoi les gens que nous voyions au bord des routes nous recommandaient de réduire la vitesse. Abaissant répétitivement l'avant-bras, la paume de la main vers le sol, ceux-ci faisaient en effet le geste qui, en Italie, signifie « ralentir ». Ce geste, je l'avais alors mal interprété. "Ils demandent un passage », m'expliqua le chauffeur. Cet épisode fonctionna soudain comme un révélateur de l'attention qu'il convient de prêter au type de malentendu que je définirais comme " culturel » et qui envahit les contextes quotidiens de la recherche ethnographique. Je me demande aujourd'hui quelle part ma peur d'un accident avait joué, au niveau inconscient, dans cette interprétation erronée ou ce malentendu : les récits de terribles accidents routiers sur ce tronçon étaient, en effet, quotidiens. Aujourd'hui, je ne puis dire si j'aurais donné une autre interprétation à ce geste dans un état d'âme différent. Il est certain que si j'avais dû me trouver moi-même au bord de cette route à devoir demander un passage, j'aurais vite appris la signification effective de ce signal.

Si cet exemple peut être défini comme un cas de "traduction intermodale » (ici, du geste au mot) ou de «transmutation », dans le cadre de la traduction proprement dite, c'est-à-dire la « traduction interlinguistique » ( $c f$. Jakobson, 1959 ; Eco, 2003), la composante culturelle intervient dans toute sa complexité, englobant toutes les dimensions de la communication linguistique, à commencer par la dimension pragmatique des actes de parole ou des pratiques langagières (speech practices) ${ }^{2}$. Quoique la langue occupe déjà une place centrale dans l'œuvre de grands auteurs comme Boas et Malinowski ${ }^{3}$, c'est dans le cadre d'une plus ample réflexion sur la spécificité de la connaissance anthropologique - et particulièrement sur le rapport entre expérience personnelle de terrain et élaboration théorique - que, dans les dernières décennies du $19^{\mathrm{e}}$ siècle, certains anthropologues ont souligné la valeur paradigmatique revêtue par la traduction.

Dans la pratique anthropologique, traduire c'est « un mouvement dynamique entre le dedans et le dehors, l'identique et le différent, la proximité et la distance »

1 Recherche universitaire menée sous la direction de Vittorio Lanternari, au cours de laquelle j'ai participé à une mission de l'équipe psychiatrique du Point G, conduite par Baba Koumaré, dans le Bélédougou (ou Beledugu, selon la graphie bamanankan), région centrale du Mali, située à environ $160 \mathrm{~km}$ au nord-ouest de la capitale et habitée en majorité par des Bambaras (Bamanan). Je suis retournée dans cette zone du Mali l'année suivante et jusqu'en 2001, pour des séjours de plusieurs mois par an, tant pour mener une recherche dans le cadre du doctorat que pour poursuivre mes travaux sur le jama (nyama), financés par des institutions italiennes et la Wenner-Gren Foundation ( $c f$. Ciminelli, 1992, 1996, 1997a, 1998a, 2000, 2004, 2005). C'est à cette période de terrain que se réfère le présent article.

2 Cette dimension prend en compte les contextes dans lesquels il est licite de parler ou, au contraire, recommandé d'observer le silence, les types de conversations dans lesquels il est possible d'affronter certains arguments, à qui et dans quelles circonstances il est permis d'adresser la parole, selon quelles modalités et quels temps, ainsi que, de manière plus générale, « les positionnements discursifs » des locuteurs (Canut, $2008: 10$ ).

3 Comme le décrit Jean-Michel Adam (1990), Malinowski (1935) avait une théorie proche de ce qui, aujourd'hui, pourrait être défini comme valeur illocutoire de certains actes de langage et valeur performative de certains verbes et énoncés (Austin, 1975 ; Searle, 1969). Kilani (1990a : 151 ; 1994 passim) rappelle également en plusieurs circonstances les indications méthodologiques fournies par Malinowski dans Coral Gardens. 
(Kilani, 1994 :14), ou, suivant les paroles de Silvana Borutti, c'est « demeurer dans la différence » entre deux systèmes symboliques (Borutti, 1981:81) ${ }^{4}$. On a souligné l'analogie que présente la construction de la connaissance anthropologique, tant au niveau de la description que de la théorisation, avec la traduction interlinguistique, et ceci tant par son "unilatéralité $»^{5}$ (Affergan, 1987 : 187) que par son caractère aporétique. Si une identité sémantique entre deux textes ne peut être en effet postulée, on ne peut donner « la » traduction d'un texte, mais plutôt (et seulement) " l'une » des traductions possibles. Le caractère paradoxal de la traduction rappelle les propos d'Evans-Pritchard sur l'anthropologie : " there's only one method in social anthropology, the comparative method - and that's impossible " (d'après Needham, 1975 : 365). Ainsi, si pour Francis Affergan (1987 : 187), traduire consiste « surtout à rendre compte des impossibilités mêmes de transiter d'un système culturel à un autre », pour Silvana Borutti (1991 : 90), traduire « est à la fois une impossibilité théorique et une pratique hypothétique d'interprétation ». Cette " pratique hypothétique » nous rappelle que - indépendamment des théories sur le relativisme, l'indétermination et l'incommensurabilité de la signification linguistique ${ }^{6}-$, les traductions toutefois existent, comme d'ailleurs la traduction intralinguistique l'atteste 7 .

Aujourd'hui, on constate un intérêt renouvelé pour la valeur paradigmatique de la traduction, même dans sa portée épistémologique, au point que William F. Hanks et Carlo Severi (2014 : 13), dans l'introduction du numéro spécial de la revue Hau intitulé « Translating Worlds », peuvent définir l'anthropologie sociale comme « non seulement l'étude des différences culturelles, mais aussi et simultanément [...] une science de la traduction : l'étude des procès empiriques et des principes théoriques de la traduction culturelle ». En fait, selon ces auteurs,

... translation does not come into play only after the translator has understood the original. It is not an ancillary re-rendering or glossing, but is itself the basis for understanding. Translation in one or another variety is always already in play, long before the overt act of re-rendering some social object into a foreign language. This is why we speak of an epistemological space of translation. (Hanks et Severi, $2014: 3$ )

Comme c'est le cas pour plusieurs concepts, la compréhension ne se donne pas sans son contraire, l'incompréhension : c'est probablement dans l'écart entre l'une et l'autre,

4 «stare nella differenza ». Sauf mention particulière, les traductions sont les miennes.

5 Ou mieux, comme j'expliquerai, par sa « directionnalité » ou « orientation ».

6 La présentation de telles théories dépasserait les limites de ce travail : pour un excursus en relation avec les problèmes de la « pratique anthropologique », voir Miceli (1982, 1990), Sbisà (1993) et Borutti (1991). Plus récemment, une brève synthèse pourrait être consultée dans Hanks et Severi (2014) ; quelques discussions à ces sujets figurent également dans le numéro spécial de Hau dirigé par ces derniers.

7 En rappelant que pour Jakobson (1959) la signification (meaning) d'une expression est simplement sa traduction intralinguistique et que pour Goodman (1978) une traduction doit se référer ou paraphraser la représentation qu'elle traduit, William Hanks (2014) affirme que la traduction interlinguistique en réalité présuppose la traduction intralinguistique - c'est-à-dire la capacité métalinguistique de chaque langue -, laquelle se révèle également centrale pour la compréhension anthropologique. 
dans les différentes formes que prend un tel écart, même au fil du temps, que se situe le lieu du travail anthropologique. C'est ce que j'entends démontrer dans le présent essai, en me fondant sur mon expérience dans un contexte linguistique bamanankan (bambara), afin de mettre en lumière différents niveaux et registres où la « traduction culturelle » tend ses pièges. Pour chacun de ceux-ci, je chercherai à analyser où se situe le « malentendu », comment il opère, comment il se manifeste, comment on peut en extraire des indications pour transformer l'incompréhension en compréhension.

$\mathrm{Du}$ reste, comme tous les termes-concepts, " malentendu " est une fuzzy notion (Kosko, 1993 ; Piasere, 2002), voisinant avec des notions tout aussi vagues (équivoque, incompréhension, méprise, etc.) apparentées par un « air de famille » et qui apparaissent dans divers « jeux de langage » (Wittgenstein, 1953). Existe-t-il une notion « prototypique » (Lakoff, 1987) du malentendu ? Il aurait été intéressant de rechercher de possibles traductions en bamanankan de ce mot, puis d'en analyser de manière contrastive la sémantique et la pragmatique, mais je n'ai pas pu le faire en son temps. C'est ici d'ailleurs, dans sa directionnalité (Asad, 1986 ; Lepenies, 1993 ; Hanks, 2014), voire dans son orientation (Ciminelli, 1998a, 2005), que réside le premier piège de la traduction culturelle. Ici, nous ne nous référons pas seulement au fait linguistique consistant en l'existence d'une langue source et d'une langue cible, mais à la dynamique sujet-objet, de nature nécessairement asymétrique, que chaque acte de traduction pose en soi. Comme le soulignait Talal Asad (1986 : 158) : " all successful translation is premised on the fact that it is addressed within a specific language, and therefore also to a specific set of practices, a specific form of life $»$. De plus, chaque traduction, qu'elle soit réussie (successful) ou non, est non seulement dirigée vers une culture mais également orientée par la culture d'appartenance du sujet qui traduit (Ciminelli, 2005 : 23). Il n'est, en effet, jamais indifférent de savoir « qui est le sujet qui traduit, ainsi que de et vers quelle langue on traduit », puisque le traducteur est

le sujet qui effectue une transcription particulière de significations, établit les démarcations et fixe les limites, détermine (et souvent surdétermine) l'objet de la traduction. Une telle problématisation semble incontournable dans la connaissance anthropologique, où l'opposition sujet/objet correspond à la dichotomie nous/autres et implique des questions de pouvoir. (Ciminelli, 1998a : 154)

Talal Asad ${ }^{8}$ a été le premier, en 1986, à constater que lorsqu'un sujet traduit depuis l'une des langues « fortes » ou dominantes (celles des pays occidentaux ex-coloniaux) vers l'une des langues « faibles » ou subalternes (celles des pays du tiers et quart monde $)^{9}$, nous sommes face à un cas d' « inégalité des langues » (Asad, 1986: 155 ss.) : « Western languages produce and deploy desired knowledge more readily than Third

8 En reprenant une suggestion de Rudolf Pannwitz, mentionnée par Walter Benjamin (1969) et en faisant une critique à Ernest Gellner (1970) sur la prétention de fournir le « real meaning » de ce que disent les Berbères.

9 Nelson Graburn (1976) a nommé « quart monde » (Fourth World) les peuples indigènes, ou autochtones, dont le territoire se trouve au sein des frontières nationales et de l'administration bureaucratique des pays du premier, deuxième et troisième monde. 
World languages do. (The knowledge that Third World languages deploy more easily is not sought by Western societies in quite the same way, or for the same reason.) » (ibid.)

De ce fait, à cause surtout de la plus grande capacité de manipulation mise en œuvre par les pays occidentaux dans les relations politico-économiques, les langues « faibles » subissent, selon Asad, " a transformation (lexical, grammatical, semantic) ${ }^{10}$ that is far more radical than anything to be identified in European languages » (Asad, 1986 : 158). Le sociologue Wolf Lepenies abondait dans ce sens en se référant spécifiquement à la culture scientifique occidentale, soulignant que celle-ci « se conçoit elle-même comme universelle ; elle exige des traductions, mais ces traductions peuvent se réaliser dans une seule direction $»^{11}$ (Lepenies, 1993 : 12 ; voir aussi Hanks, 2014).

En outre, une traduction orientée de la culture forte vers la plus faible tendra très probablement à négliger l'espace sémantique dans lequel se situera le lexème choisi comme traduction, ce qui entraînera des résultats insatisfaisants. En effet, il est désormais évident que, si au niveau lexical intralinguistique la signification d'un mot dépend du sens des mots appartenant au même champ sémantique, au niveau interlinguistique il se peut que, dans une analyse contrastive, des champs sémantiques entiers puissent ne pas se trouver en correspondance : « from a contrastive point of view, whole semantic fields - being language-specific - will not be matchable between languages » (Ivir, 1977 : 170). Evidemment, d'un point de vue anthropologique, cette spécificité n'est pas seulement linguistique, mais aussi culturelle.

J'ai tenté ailleurs de montrer les conséquences négatives provoquées par ces traductions orientées. Le terme français de «paludisme », traduit en bamanankan par sumay $^{12}$ dans le cadre de la formation sanitaire conduite au Mali dans les années 1980 sans avoir tenu compte du plus vaste système santé/maladie local, a provoqué un « abus de diagnostic d'accès palustre » de la part des agents de santé, comme cela avait été également le cas dans d'autres pays (Bonnet, 1986, in Roger, 1993 : 85). L'usage de ce terme entraîna aussi, dans certains cas, des choix thérapeutiques erronés de la part des « matrones », les sages-femmes des centres de santé (Ciminelli, 1998a, 2005). Ces erreurs thérapeutiques étaient imputables à la superposition injustifiée, voire abusive, des catégories biomédicales de paludisme et de tétanos néonatal. En effet, au cours de la même période, l'apparition du kons, la maladie de l'oiseau ${ }^{13}$, était indiquée comme l'une des possibles conséquences d'une sumaya négligée chez les petits enfants : toutefois,

10 Cet aperçu a été repris récemment par William Hasch, qui introduit le concept de « commensuration » comme une sorte de traduction interlinguistique qui se base sur la capacité métalinguistique que possède chaque langue (Hanks, $2014: 30$ ).

11 «Questa cultura scientifica concepisce se stessa come universale ; esige traduzioni, ma queste traduzioni possono realizzarsi solo in una direzione ».

12 Sumaya comme état d'altération est souvent traduit en bamanan par refroidissement ou grippe. Sumaya (suma, fraicheur humide, -ya, suffixe d'abstraction) qualifie le temps typique de l'hivernage (samiya) ou saison des pluies, particulièrement propice au paludisme. Toutefois l'air suma n'est pas un mauvais air (en italien, mal'aria), et les significations de suma sont traditionnellement positives, comme témoigne par exemple le souhait « k'a suma i kons » (que ceci [la nourriture] devienne suma en toi), par lequel répond la cuisinière à celui qui apprécie sa cuisine (" $i$ ni gwa! » : toi et le foyer !), ou encore sa signification de « calme », où suma est utilisé en relation avec les « maladies de la tête » (voir infra).

13 Kon signifie oiseau : la cause de la maladie kənっ, ou konsbana (bana, maladie), est le jama (Ciminelli, 2004) que l'engoulevent transmet même en survolant la case de la femme qui a accouché. 
désignant également les manifestations de la maladie homonyme, «kons » recouvre en bamanankan tant les convulsions dues aux attaques fébriles d'origine malarique que les spasmes causés par le tétanos néonatal (Ciminelli, 1998a : 156-166, 2005).

Si c'est uniquement dans nos sociétés que l'on peut "légitimement isoler » un système médical, comme l'affirmait Nicole Sindzingre (1984 : 100), il est cependant possible de se référer de manière plus générale à un système santé/maladie (Ciminelli, 1998a), celui-ci étant envisagé comme la/une structure d'oppositions hiérarchiques (Dumont, 1979, 1983, 1988) opposant la santé à la maladie - mieux, aux maladies vues comme des altérations, différences ou négations d'une norme qui peut paraître naturelle, mais qui est plutôt « imposée » à la nature (Canguilhem, 1966).

Sur le plan logique, les oppositions hiérarchiques sont analogues aux oppositions privatives ${ }^{14}$ et à la « relation d'exception » de Giorgio Agamben ${ }^{15}:$ le terme non marqué, qui revêt une signification et une valeur de normalité (qui est également normative), n'est pas perçu dans ses "valeurs spécifiques ", sauf quand il est en position de contraste. En synthèse, la caractéristique des oppositions privatives et/ou hiérarchiques (exemple : homme $\sim$ femme) est l'ambivalence du terme non-marqué (homme), dont la « signification restreinte » ou « valeur spécifique » (être humain de sexe masculin) ne se manifeste pas là où il fonctionne comme légitime représentant de la totalité (homme comme être humain $\sim$ non humain ou inhumain), apparaissant uniquement dans des contextes où l'opposition est pertinente (homme comme être humain de sexe masculin $\sim$ femme). Dans le premier cas, dit de " neutralisation de l'opposition », l'on a donc affirmation de $A \sim$ non affirmation de $A$, dans le second, affirmation de $A$ $\sim$ affirmation de non-A. Dumont (1983 : 263) ajoute aux oppositions privatives une dimension supplémentaire, en notant que dans les oppositions asymétriques dont il fait état, " l'englobement du contraire » indique une valeur « hiérarchique » qui n'apparaît pas là où l'on considère l'opposition comme une simple « distinction à l'intérieur d'une identité " (comme c'est le cas des oppositions binaires de l'analyse structurelle lévi-straussienne, qui sont de type qualitatif et symétrique) ${ }^{16}$. Même l'opposition

14 Initialement élaborées dans un contexte phonologique par Trubetzkoy, reprises dans un cadre sémantique par Jakobson et utilisées comme instrument d'analyse sémantico-cognitive par Greenberg et d'autres auteurs (Ciminelli, 1998a : 128 ss.).

15 Comme l'indique le même philosophe italien dans le passage dédié au concept de Popolo/popolo (Agamben, 2005 : 198-199).

16 L'englobement du contraire peut être facilement reconnu dans un certain « nous » qui a une prétention universaliste, mais qui révèle son contraire et, par conséquent, sa valeur spécifique (nous comme opposés aux autres, inférieurs) quand on est « contraint » à marquer l'exception. Ce dispositif fonctionne dans divers domaines, comme je l'ai expliqué ailleurs. Il est par exemple reconnaissable dans le concept " culture-bound », introduit dans les années 1960 par Pow Meng Yap pour caractériser les syndromes psychiatriques des autres, les nôtres ayant ainsi l'air d'être culture-free et donc assumant une fausse valeur universelle, dont on ne perçoit pas la valeur (culturelle et anthropologique) spécifique. Les culture-bound syndromes continuent du reste à dénoter les mêmes référents des syndromes anciennement appelés « exotiques » par Emil Kräpelin, et diffèrent de ceux-ci seulement en ce que Yap substitue au facteur étiologique collectif « race », le facteur « culture » (Ciminelli, 1997b; 1998a ; 1998b). Pour donner un autre exemple, ce dispositif est reconnaissable dans la nécessité de caractériser « un certain » art contemporain comme aborigène, africain, natif, etc., le nôtre étant de l'art contemporain tout court, non marqué (Ciminelli, 2013 ; 2016), comme déjà un art primitif avait été distingué de l'Art avec majuscule (Ciminelli, 2008a). 
hiérarchique santé/maladie, au sens d'« un ensemble ou système structuré par ses oppositions internes » (Dumont, $1988: 25)$, est donc en relation avec un " tout» hiérarchiquement supérieur (Dumont, 1983 : 243) - la vie - et cesse d'être pertinente (et d'avoir du sens) avec la mort. L'absence de santé est en effet une condition remplie tant par la maladie que par la mort. Ainsi, le terme non marqué (santé), dans une position de neutralisation (quand " santé " est opposée $\grave{a}$ la mort et non $\grave{a}$ la maladie) finit par assumer une connotation générale de normalité et de naturalité, dont on ne perçoit pas la signification restreinte ou la valeur spécifique ; la santé est en effet la norme de la vie (ou imposée à la vie), la norme « naturelle ».

Une multiplicité de termes s'opposent donc à un seul, pourvu d'une valeur positive : celui que désigne l'"être en bonne santé »-en bamanankan : kenc. Si les maladies sont nombreuses, la santé est, en effet, unique. Les « valeurs spécifiques » de kene apparaissent seulement en explorant les oppositions qui structurent intérieurement le domaine sémantique du concept, et ceci au moyen d'une analyse différentielle, qui se sert de manière contrastive de la langue française (Tab. 1).

Il est ainsi possible de faire émerger la richesse symbolique d'un domaine sémantique dans lequel même les métaphores utilisées par les guérisseurs traditionnels, comme nous le verrons, plongent leurs racines anciennes et nouvelles (Ciminelli, 1997a, 1998a, 2000).

Dans le champ sémantique de $k \varepsilon n \varepsilon$, les divers registres qui font appel aux articulations du sensible caractérisent la santé comme une qualité spécifique de la nature, dotée d'une valeur positive. Si dans le tableau émerge tout de suite, en traduction, le registre de l'opposition chaud/froid, lequel est généralement rapporté au paradigme hippocratique de l'équilibre des éléments, transmis par les Arabes, les termes originaux bamanankan désignent une plus riche constellation de significations.

Par exemple, même si $k \varepsilon n \varepsilon$ ne peut être considéré comme un vrai chromonyme, il donne toutefois naissance à un terme dérivé, binkene : vert clair, « la couleur de l'herbe (bin) fraîche $(k \varepsilon n \varepsilon) »$. Si l'on dit que l'herbe est $k \varepsilon n \varepsilon$, cela signifie aussi qu'elle est « verte », autrement elle ne serait pas « fraîche » - « here there are language games that decide these questions » (Wittgenstein, $1977: \S 6$ ).

Des autres oppositions émerge une sorte de concept thermodynamique de la santé, en particulier quand entre en jeu le terme kalan - une chaleur différente de celle qui afflige le corps des fiévreux (gwan.ts, chaleur.sujet), une chaleur qui, comme en physique, est aussi mouvement. Cette variété de chaleur, kalan, qui également dans le registre visuel indique le dynamisme (kalan, bariolé, zébré), désigne certains types de folie « agités » ( fa kalaman, folie chaude), en opposition avec ceux que l'on qualifie de «calmes » ( $f a$ sumalen, folie fraîche). Keifa D., le grand connaisseur (soma) et guérisseur de « maladies de la tête » (kunkolo.bana, tête.maladie), nous précise la portée significative de certains termes relatifs aux troubles mentaux - jawuli, hakiliwuli, kunkolowuli, hakiliwuli, jawuli ${ }^{17}$ - avec une sorte de übersichtliche Darstellung (« une représentation

17 Ces termes sont des synonymes composés par le verbe wuli (lever, soulever, etc.) prédiqué des composantes de la personne : la tête (kunkolo), l'esprit (hakili) et le ja, traduit par « ombre », « double », « esprit », « âme » mais aussi par « image » selon les contextes, une complexe composante « eidétique » de la personne qui mériterait un développement plus ample. Le trois synonymes s'opposent à l'état de santé mentale, conceptualisée par l'état de stabilité (sigi, s'assoir) des mêmes composantes (kunkolosigi, hakilisigi, jasigi). Voir aussi infra. 
claire et simple », Wittgenstein) de la relation entre chaleur et mouvement : « le ja se lève (wuli) s'il s'est trop chauffé (goniyara kojugu) : quand tu mets l'eau dans un récipient, elle est ferme (sigi), mais si tu mets le récipient sur le feu, l'eau bout (wuli), se lève ou se soulève $(\text { wuli })^{18} \gg($ Ciminelli, 1998a : 225). La relation entre chaleur et mouvement à travers laquelle se conceptualise l'état de normalité/anormalité, autrement dit l'état d'altération par rapport à la norme naturelle de « fraîcheur-santé », est aussi exprimée par le verbe goniya, qui signifie « chauffer » (sens propre et figuré) ou « accélérer ».

De ce réseau de significations émerge aussi un autre modèle, assez diffus d'ailleurs : celui de l'équilibre, qui non seulement renvoie au système gréco-romain des quatre éléments, mais aussi à notre common sense (où, par exemple, un déséquilibré est un aliéné), et qui voit dans l'excès et la carence deux principes pathogènes basilaires (Roger, 1993 : 101 sq.).

C'est finalement dans le registre alimentaire que se déploient les métaphores cognitives ${ }^{19}$ relatives à la santé. Ainsi, l'une des formules utilisées pour manifester son bien-être ou son état de malaise est ne fari ka/man di ne na: littéralement, " mon corps est/n'est pas bon (agréable) pour moi ». De même, un corps kumu «acide » est un corps affligé, souffrant de douleurs (Diaouré, 1992). On retrouve le même modèle alimentaire sur le plan des composantes de la personne impliquée dans les états de santé mentale. Ainsi, un « hakili bon, agréable » (hakili ka di) est, ou désigne, l'esprit d'une personne dont on apprécie les attributs intellectuels (intelligence, mémoire, etc., selon les contextes), lesquels attributs sont dépréciés dans le cas de hakili pas bon (man di) ou mauvais au goûter ( $g o$ ), ou encore contaminé, sale (nəgo), et se perdent dans le cas de hakili abîmé ou " gâté » (tincn). Il est opportun d'ajouter que le sens positif de $k \varepsilon n \varepsilon$ comme « sain », qui qualifie également les aliments « frais » (salubres, salutaires), s'estompe, voire s'efface, quand l'opposition santé/maladie n'est plus pertinente, à savoir en relation avec la mort, comme nous l'avons vu ci-dessus : dans ce cas, un corps (un cadavre) kene est un cadavre « frais » seulement au sens de " récent », par rapport à un autre kors, qui signifie « vieux ».

18 Dans le dictionnaire de Bailleul et al. (2011-2014), le verbe wuli dans sa signification de « bouillir » est enregistré séparément du lexème polysémique wúli (se lever, faire lever, soulever, etc.), or, le témoignage de Keifa D. démontre au contraire qu'il s'agit du même lexème. La même réflexion peut être appliquée au qualificatif kálan, dont le sens « bariolé » etc. devrait être réadmis dans le cadre de la polysémie du lexème kálan (« chaud, rapide »).

19 Les métaphores cognitives (Lakoff et Johnson, 1980a ; Lakoff, 1987, 1993) ou " conventionnelles » (Lakoff et Johnson, 1980b) correspondent à des " façons de dire » que nous employons couramment dans la vie quotidienne, sans d'ailleurs remarquer leur qualité métaphorique. Par exemple, on parle du temps en termes d'espace (« les vacances sont passées », « Pâques est arrivé », etc.), ou de la quantité et de la valeur en termes de verticalité (supérieur/inferieur). En bref, ces métaphores, le plus souvent ancrées dans l'expérience vécue du corps, reposent sur la projection, ou « mapping », de la structure conceptuelle d'un domaine « source », normalement concret (par exemple, le schéma de la verticalité dérive de l'expérience de la gravité), sur un domaine « cible », normalement abstrait ou plus abstrait (dans notre exemple, la valeur ou la quantité), de manière qu'on puisse le maitriser cognitivement. 
Tableau 1 : Analyse différentielle du concept bamanan de santé

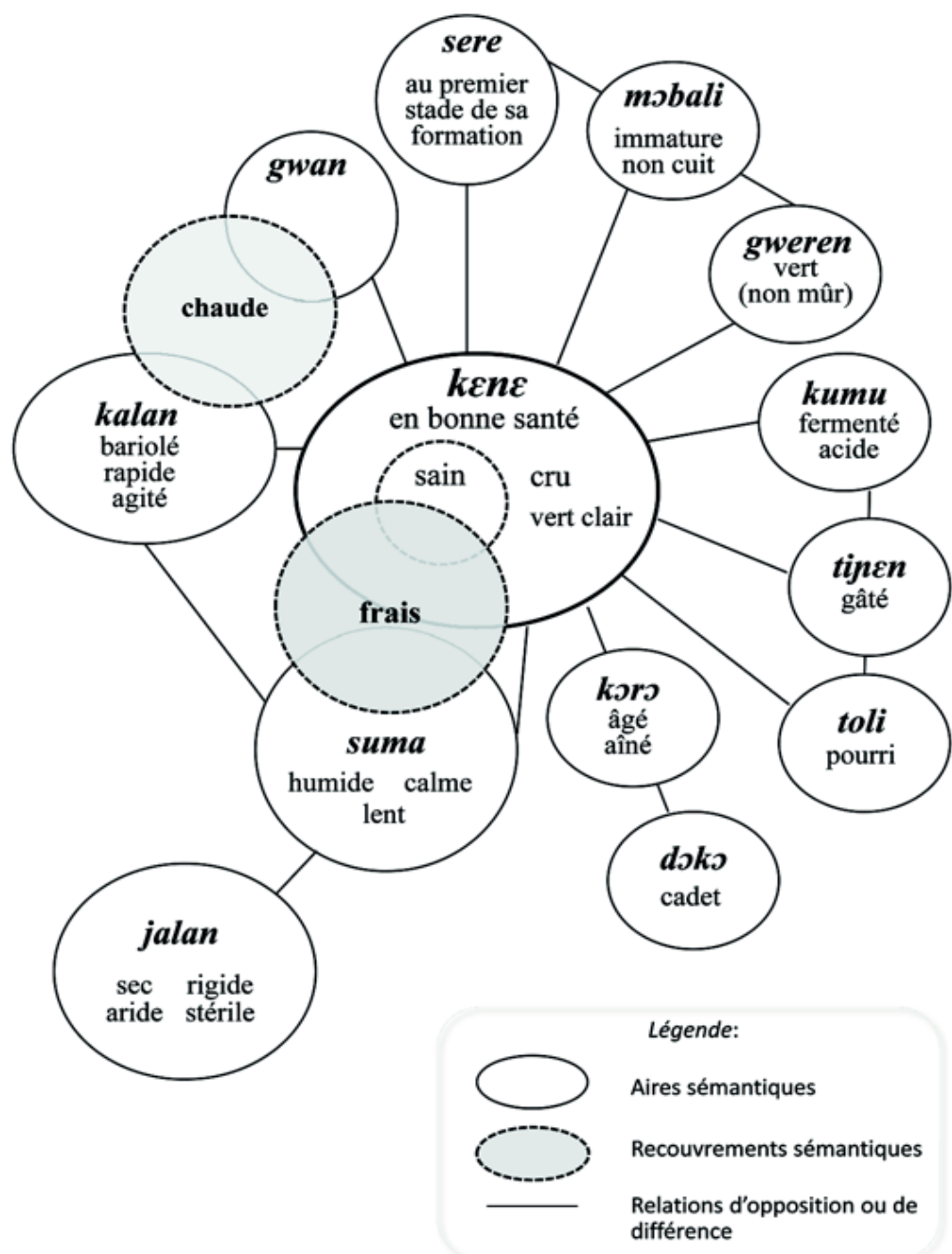


Une variante de malentendu ou d'incompréhension d'ordre culturel, qui fut signalée pour la première fois par Roger Keesing (1984), concerne justement le risque d'interpréter certaines « façons de dire » (« ways of talking ») comme des preuves de visions du monde radicalement différentes des nôtres, alors que parler du passé et du futur, d'émotions, de causalité, etc., est « inévitablement métaphorique, autant chez nous que dans d'autres populations » (Keesing, 1987 : 168) : les métaphores auxquelles Keesing se réfère sont surtout de nature cognitive. Reconnaître une métaphore, sa qualité poétique ou cognitive, n'est pas possible, évidemment, sans connaissance de la langue dans laquelle elle est formulée : là où dans des cultures autres, les données linguistiques semblent renvoyer à des modèles radicalement différents - et exotiques - de cerner et construire la réalité, il est avant tout nécessaire de chercher à comprendre telle ou telle expression en reliant les termes qui les composent dans la chaîne sémantique plus ample de la langue locale. Ainsi, on pourrait être d'accord avec Hanks (2014 : 21) quand il affirme que la traduction - entendue comme tentative, de la part d'un ethnographe ou d'un linguiste, d'expliquer « la cohérence et la signification d'un concept appartenant à une culture lointaine » - «n'est pas seulement question de redécrire une forme culturelle, mais, premièrement, de la comprendre ».

Williams Hanks touche à un autre aspect, se référant à la traduction et à son orientation, qui renvoie à une forme différente de malentendu ethnographique. Souvent, dans ce procès qu'il appelle de " commensuration $»^{20}$, « la traduction cesse d'être une relation binaire entre deux langues, et devient une relation triadique entre deux langues qui sont mises en rapport par un registre de néologismes, dans l'une des langues (ou bien dans les deux) » (Hanks, 2014 : 35). Or, le plus souvent c'est normalement la langue la plus « faible » (d'après Asad) qui subit la néologisation, plutôt que le contraire, de sorte que dans cette « relation triadique » l'échange linguistique bidirectionnel apparaît comme « inévitablement asymétrique » (Hanks, $2014: 30)^{21}$.

Un problème de relation «triadique » entre français et bamanankan, et donc un problème de néologismes, fut aussi à l'origine de mes premières recherches en milieu bambara (Ciminelli, 1992).

Le psychiatre transculturel, Raymond Prince (1960), avait identifié parmi des étudiants nigérians un nouveau syndrome culture-bound ${ }^{22}$, le "brain-fag », dont la symptomatologie semblait correspondre au « surmenage mental » ou « surmenage intellectuel », sensiblement répandu dans le milieu scolaire et universitaire malien (Koné, 1978 ; Bastien, 1988 ; Diakité, 1989). L'ethnologue Christine Bastien (1988) avait indiqué que ce "surmenage » était la traduction du mot kalankèbana, c'est-à dire «maladie du savoir » ou "maladie de l'étude » (kalan.ke.bana, étude/savoir.

20 Par « commensuration », Hanks (2014:30) indique une variante spéciale de traduction interlinguistique qui se base sur la capacité métalinguistique de chaque langue.

21 Ainsi, on ne saurait être totalement d'accord avec Hanks quand il affirme que « It is the target language into which one translates that ultimately constrains the process », du fait, justement, que « Historical examples of languages changing through intertranslation abound, but the clearest are found in colonial contexts in which authoritative texts in a dominant language are translated into a subordinated language, for this process inevitably alters the semantics and pragmatics of the subordinate language » (Hanks, $2014: 18-19)$.

22 Voir la note 16. 
faire.maladie). Ce mot lui semblait être « une catégorie traditionnelle probablement antérieure à la colonisation, reprise et adaptée par les jeunes acculturés pour exprimer leurs conflits, et maintenant unanimement reconnue par les praticiens de tous ordres et par le public » (Bastien, 1988 : 201). Le mot kalankèbana contrastait toutefois avec les traductions bamanankan de "surmenage mental » fournies par le médecin malien Djigui Diakité (1989) et, comme je l'ai vérifié par la suite, était également inconnu de mes informateurs. Ils connaissaient par contre d'autres noms de maladie - parmi lesquels, en premier lieu, hakiliwuli, jawuli, kunkolowuli - que tant Bastien que Diakité mentionnaient en rapport avec le surmenage des étudiants (Ciminelli 1998a). Au terme de mes recherches, le kalankèbana s'est révélé être un néologisme bamanankan introduit par le marabout et guérisseur Moussa D., qui avait traité dans un contexte urbain une vingtaine de «surmenés » et qui était le seul guérisseur à en user expressément (Bastien $1988: 102,199$ seg.) ${ }^{23}$. Il s'agissait donc d'une traduction orientée du français au bamanankan, plutôt que le contraire (Ciminelli 1998a : 185-186). Attribuant une direction, voire une orientation erronée, à ce qui était, effectivement, une traduction, Christine Bastien a été donc induite à une mésinterprétation, c'est-àdire à une incompréhension.

C'est toutefois dans la relation interlinguistique « sur le terrain » que l'incompréhension intervient dans toute son étendue : du malentendu à ce qui peut être interprété, selon qu'on le juge volontaire ou non, comme une manipulation ou une erreur.

Rupert Stasch (2014) s'est récemment intéressé au «pouvoir de l'incompréhension » dans une analyse qui témoigne bien de la présence d'une double orientation culturelle, de nature toujours asymétrique, dans l'interaction entre touristes étrangers et Korowai de Papouasie-Nouvelle-Guinée. Stasch prend en considération tant les contextes pragmatiques que les vocables qui y sont utilisés ${ }^{24}$, ainsi que les manipulations de la part du guide-interprète, paradoxalement appelé « chef des touristes » par les Korowai, en raison de la valeur de subordination que revêt dans cette société le " prêter écoute » à une personne. Son analyse fait apparaître une synergie entre l'incompréhension linguistique et l'attente primitiviste occidentale (Stasch 2014 : 85), certes non limitée au seul domaine touristique ${ }^{25}$ et que les manipulations de la communication par les interprètes visent par ailleurs à satisfaire. Un exemple d'illustration pourrait être celui des sous-titres du film Path to the Stone Age de 2010 : censé traduire correctement en korowai la demande « Have you ever seen white men ? », l'interprète déclare en réalité à ses interlocuteurs : " Say 'No, none.' Say 'None at all.' Say 'I have not seen whiteskinned people, this is the first time' » (Stasch 2014 : 83-84).

En effet, quand la communication interculturelle est modulée par un interprète, les possibilités de malentendu (lato sensu) se multiplient. A ce sujet, je voudrais revenir sur l'un des premiers entretiens que j'ai enregistrés au début des années 1990 dans

23 L'ethnologue avait aussi annoncé au début de son ouvrage que « la somme du matériel recueilli auprès de Moussa D. contraste avec les données provenant des treize autres informateurs » (Bastien, $1988: 22$ )

24 Voir, en particulier, l'utilisation de manop (Stasch, 2014 : 79).

25 Par exemple, dans les domaines de l'art et du patrimoine culturel (Ciminelli, 2008a, 2008b, 2009, 2011, 2016). 
le Bélédougou, afin de le réexaminer à la lumière d'une nouvelle traduction du texte bamanankan.

Cette version, que je définirais comme une « contre-traduction », même s'il ne s'agit pas en réalité d'une traduction inverse (back-translation), a été effectuée par un collègue anthropologue et archéologue, le professeur Samou Camara. Dans les tableaux suivants, la conversation sur le terrain (colonne de droite), qui se compose d'un dialogue

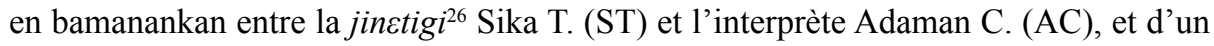
dialogue en français entre l'interprète $\mathrm{AC}$ et moi (MLC), sera mise en contraste avec cette nouvelle « contre-traduction » du texte bamanankan (colonne de gauche).

Il en résulte deux textes assez différents, dans lesquels l'enchainement des questions et des réponses suit souvent des logiques différentes, parfois inconciliables, avec des effets qui couvrent toute la gamme qui va de l'inexactitude au malentendu, jusqu'à l'équivoque et aux aspects comiques.

A ce moment-là, je n'étais pas en mesure de suivre le dialogue. Je comprenais néanmoins certains mots isolés ; quelquefois, je croyais simplement les avoir entendus (dans le double sens du vocable). Il aurait été intéressant de pouvoir reporter l'entretien dans son intégralité ${ }^{27}$, mais l'espace mis à notre disposition nous permettra d'analyser quelques extraits.

Dès les premières phases de l'entretien avec l'informateur et du dialogue en français entre l'interprète ( $\mathrm{AC}$ ) et moi (MLC), un problème de traduction s'est posé (Tab. 2).

Pourquoi AC traduit-il les paroles de la jinctigi par surmenage? Pourquoi soutientil, après ma demande, que celle-ci ait parlé de kunkolowuli, alors que ST parle seulement de kunkolodimi, maux de tête (kunkolo.dimi, tête.douleur) ? Une manipulation de la part de l'interprète, qui connaissait tout mon intérêt pour le surmenage mental, me sembla indéniable et me plongea dans l'embarras. C'est seulement plus tard que j'ai compris que sa réponse était motivée non seulement par l'intention de ne pas me contredire, par respect (j'étais tubabu, blanche, son employeur, surtout j'étais kə̀ro, plus âgée), mais aussi par l'existence d'un lien, sinon d'une identité référentielle, entre kunkolowuli et surmenage, dont les céphalées (kunkolodimi) constituent d'ailleurs le symptôme principal (Koné, 1978 ; Bastien, 1988 ; Arwata, 1984).

Croyant suivre le fil du dialogue, je demande donc si kunkolowuli et jawuli sont la même maladie. La guérisseuse affirme qu'il s'agit de « une [seule] chose » (Tab. 2).

Kunkolowuli et jawuli décrivent en effet la même évolution négative de l'état de « frayeur » provoqué par la rencontre avec les jinc : le jatike. Comme dans le susto latino-américain, c'est la perte - ou le détachement (tike, couper) - de l'ombre, la sombra, qui provoque la maladie (Polia, 1998). « Ombre » n'est du reste que l'une des traductions possibles de $j a$, une composante complexe, « eidétique » de la personne ( « double », « image », mais aussi « esprit », « âme »), sur laquelle il n'est pas possible ici de s'étendre. Face à la vision de quelque chose d'étrange et d'inattendu, « quand/si le $j a$ se lève [wuli], cela devient des maux de tête [kunkolodimi] », insiste ST : les maux de tête représentent en effet, comme l'attestent la littérature et tous mes interlocuteurs bamanan, le symptôme privilégié du jincbana, la maladie des jinc.

26 Prêtresse (jinc.tigi : djinn.propriétaire/maître de) du culte de possession jincdon (Gibbal, 1982).

27 Il a été publié intégralement mais avec une contre-traduction en italien (Ciminelli ,1998a : 243-272). 
Tableau 2

Traduction du texte banankan

ST. Certains deviennent maigres [keretete, secs secs], eh bien, si ceux-ci viennent, je les soigne. Bien, certains ont des maux de tête, leur maladie c'est les maux de tête, s'ils viennent, je les soigne. Douleurs au ventre, au ventre, certains ont mal au ventre à tel point qu'ils se tordent [tournent], s'ils viennent, moi je les soigne. Les maladies que je soigne sont celles-ci.

\section{Dialogue sur le terrain}

ST. Ka dow ja keretete, ayiwa, n'olu nana, ne bi olu furake. Ayiwa, kunkolodimi be dow la, u ka bana ye kunkolodimi ye : n'olu nana, ne b'u furake. Konodimi, kono, dow kons b'u dimi fo u bi yelema yelema : n'olu nana, ne b'u furake. Ne konila bana furake ta de ye olu ye.
AC. Elle dit maintenant... On a posé la question : quelles sont les maladies qu'elle traite ici ? Elle a cité beaucoup de maladies, par exemple elle a commencé à dire d'abord concernant les jine vous connaissez les jine déjà si quelqu'un en est atteint très gravement, qui ne peut pas se tenir lui-même, donc si ce dernier est venu directement elle le traite. A part ça, elle a dit aussi les maux au ventre, ensuite quelqu'un qui a mal à la tête. Ensuite le surmenage, qui traite tout ça.

MLC. Elle a employé quel mot pour dire surmenage?

AC. Bon, elle a employé que si quelqu'un par exemple, il va ailleurs et raconte des histoires inutiles, si on l'amène elle traite.

MLC. Et ça c'est le kunkolowuli. Elle a dit kunkolowuli?

AC. Oui, elle a dit kunkolowuli.

MLC. Est-ce qu'elle peut me dire si kunkolowuli et jawuli c'est la même maladie?

AC. Dis, maintenant, elle te demande : kunkolowuli et jawuli sont-ils des maladies du même genre ?
AC. Ko sisan, a b’i nininka : kunkolowili ani jawuli, yali bana suguya kelen de don wa?
ST. Il s'agit de la même [kelen, ST. $A$ be de ye kelen ye. un/une] chose.
AC. Elle a dit que ce sont deux maladies égales.

Il existe une autre formulation du jawuli, qui recourt au terme normalement traduit par « esprit », c'est-à-dire hakili : expression d'origine arabe, dont le sens est plus large - référant aussi à l'« attention », à la «mémoire », à la « pensée », à la « réflexion »- et que l'on retrouve chez les Dogon (hakilé), les Songhay (lakkal) et les Peul (hakkillo). Il résulte de mes données de terrain, comme l'affirmait le docteur Diakité (1989; Ciminelli, 1998a : 55 ss.), que kunkolowuli, jawuli et hakiliwuli - les « catégories nosologiques 
traditionnelles » par lesquelles on traduit le surmenage mental (v. supra) - sont à leur tour prodromique ${ }^{28}$ d'un état de « sortie » (bo) de la condition (et apparence, sawura) de personne humaine et, par conséquent, de la folie ( $f a$ ) (Ciminelli, 1998a : 200).

Le même mécanisme d'implication de significations, plus que de manque de véridicité dans la traduction d'AC, se retrouve dans un autre passage de l'entretien (Tab. 3).

Selon la version de AC, la jinctigi a eu, lors de la rencontre avec le jinc en brousse, des «palpitations » : nonobstant la confirmation que je reçois de AC, la guérisseuse n'a pas parlé du cœur (dusu, dusukun) ni de ses palpitations, normalement traduites par dusukun yere yere, ou dusu pan pan. Toutefois, ici aussi se dégage un rapport logique, puisque le jatike (effroi, frayeur) provoqué par la rencontre avec le jinc s'accompagne d'altérations du battement cardiaque, et les palpitations constituent d'ailleurs l'un des symptômes de l'anxiété, pareillement traduite par jatike dans le cadre psychiatrique (Koumaré et Coudray, 1986). Dans d'autres témoignages, dusuwuli apparaît, du reste, comme l'un des synonymes de jawuli, hakiliwuli, kunkolowuli.

Il est intéressant de noter l'emploi du terme " traduire » par l'interprète, pour signifier le lien entre le jinè et la guérisseuse : « je peux dire qu'elle suit ce jinc-là, c'est le jinc qui ... traduit toutes ces choses à elle », explique-t-il avec un instant d'hésitation, à la recherche du mot juste. Aujourd'hui, cet instant d'hésitation résonne en moi comme un écho de mes tentatives répétées d'établir un protocole correct de traduction. « Ne pas interpréter, mais traduire littéralement », telle était plus ou moins la substance de la question, ou, en d'autres mots, ce que je demandais à mon interprète. Mais « traduire », « interpréter » et « comprendre » ne sont en définitive que des vocables à traduire, interpréter, comprendre ... Les " déformations " que la traduction de AC apportent au texte (du français au bamanankan, ou du bamanankan au français) ne sont certainement pas moins « informatives » par rapport à ce que je peux comprendre de la « contre-traduction », bien qu'alors je n'étais pas en mesure de le reconnaître ... encore moins de l'apprécier. Dans ce même extrait (Tab. 3), se dégage par exemple la circonspection et la délicatesse que manifeste AC par rapport aux demandes sur des sujets complexes, comme précisément celles relatives aux modalités d'acquisition du savoir, des questions que $\mathrm{j}$ 'avais formulées de manière tellement « directe », au risque de manquer d'éducation ou pire : "Ces questions ne sont pas gênantes? Tu les acceptes toutes ? Ce n'est pas un dérangement ? Il s'agit de connaître, nous nous écoutons... ». 


\section{Tableau 3}

AC. Ces questions ne sont pas gênantes? Tu les acceptes toutes? Ce n'est pas un dérangement [baasi te] ? il s'agit de connaître, nous nous écoutons...
AC. Ninikali nin baasi te koni, $i$ bi son a bec ma, baasi te, ko don de don, an be nogon lamen...

\begin{tabular}{ll}
\hline ST. Bien sûr! & ST. Aws! \\
\hline AC. C'est-à-dire, elle a dit une & AC. C'est-à-dire, a ye kuma do \\
autre parole $[$ kuma $]$. Elle dit de te & fana fo sisan, ko k'i ninika ala : \\
demander ceci : le savoir qui est & donniya min be e yercla sisan, \\
en toi maintenant, comment as-tu & yali donniya e y'a soro cogodi? \\
pu acquérir ce savoir? &
\end{tabular}

ST. Ce savoir ? Je n'étais en possession de rien [aucune terre] dans la case du monde, dans le séjour dans le monde. Mon mari est mort très tôt, j'ai de touts petits enfants, je ne savais même pas comment les nourrir, je ne pouvais même pas me procurer de pagne à attacher. Bon, je suis allée en brousse pour déterrer des racines sauvages. Quand je suis allée en brousse pour déterrer des racines, il s'est passé comme si tu dis une image, un miroir; une sorte de miroir s'est installée [sigi] sur mes yeux, me montrant et montrant des choses [jirana]. Ces choses montrées [jirana] se sont arrêtées. Je me suis préparée pour retourner à la maison. Je n'ai rien trouvé ce jour-là, je n'ai même pas pu gagner le repas du soir de mes enfants. Je suis allée ainsi me coucher dans ma case. Cette certaine chose, c'est elle qui est venue me donner comme ça toutes ces connaissances. Bon, quand quelqu'un tombe malade, s'il vient me trouver, je dis « ça c'est la maladie de jine », je vais en brousse, je coupe les feuilles, je coupe le remède [fura, feuille] correspondant et cette personne guérit. Quand quelqu'un vient, qu'il s'agit de la folie, je vais en brousse, je vais couper le remède correspondant et la personne guérit. Les personnes que j'ai guéries sont nombreuses, hein ! Même si tu les mets dans ma cour, elle ne pourra pas les contenir. De ceux qui se roulent par terre à ceux qui tremblent, la plupart je les traite.
ST. Donniya nin ? Dugu kolo foyi foyi de, kun te ne bolo, dine so kons, dine lategero. Ne ce de sara josna, denw misenmanin miscnmanin be n'bolo, hali $u$ balo cogo kun te n'bolo, hali nka fini siri ta kun ta sors. Bon, ne taalen nana seni kungo kons. N'taalen nana seni kungo kons, a kera de k'i ko ja, jafile : jafile d'u nana sigi n'ye na sa, ka jirana jirana jiranaw ke n'na ten. Bon, jirana jirana nunu kelenna, nininna ka na so. N'man nana soro, hali nka denw suro fana ma sory a donna; o ne nana n'da su kons sa. A fen masuma in, a le de nana ka na faamuyali nunu di ne mo ten. Bon, ni min banana, n'o nana n'ba fo " nin ye jinsbana ye », n'bi taa kungo kono, n'bi tu tike, o fura tike, o bi kencya, nin min nana n'o ye fa ye, n'bi taa kungo ksno, n'bi taa o fura tike, o bi kencya. Ne yere ka mogo kencya kencyalen minnu o ka ca de! Hali ni y'u ken'ka kurun kons yan, a te u te kun a la, ka ta min binibana binibana kuru kara kuru, a bes, a caman ye, ne ka furake de ye. 
AC. Bon maintenant, ce qu'elle raconte sur sa connaissance même, elle dit que bon, elle n'avait rien auparavant, elle n'avait rien. Quand elle était jeune, bon, son mari est décédé très longtemps, en ce moment, les enfants étaient très petits. Au Beledugu ici, quand la famine vient, dans la brousse, on part creuser une sorte de tubercules qu'on appelle nana. On mange ça si on n'a rien à manger. Le moment qu'elle a été, bon, les enfants étaient trop petits. Bon, vraiment elle a eu des palpitations dans la brousse.

MLC. Dusukun?

AC. Oui, dans la brousse quoi. Maintenant elle même, il y a une sorte de miroir, miroir qui est venu directement à lui. Et elle a vu beaucoup de choses là-dedans. Donc le moment qu'elle est venue, elle dormait un peu, elle a rêvé - les rêves, quoi - maintenant elle a vu une personne qui lui a raconté tout : s'il a ceci, cet arbre le soigne, s'il y a cela, comme ça. Donc sa connaissance vient de ça. Donc, je peux dire qu'elle suit ce jinc-là, c'est le jine qui ... traduit toutes ces choses à elle.

En en dépit de sa délicatesse dans les questions, les traductions de AC sont souvent inexactes. La " vision » du jine par ST, c'est-à-dire la rencontre survenue en brousse entre le jinc et la guérisseuse est, par exemple, traduite par « rêve ». Dans mes tentatives de revenir sur la question, je ne reçois qu'une confirmation de la part de $\mathrm{AC}$ - mais seulement de lui, évidemment. Ou encore, de l'expression « moyens de divination » que j'utilise pour contrôler une information, AC traduit seulement « moyens », par baara (travail) : ces vocables, « moyens » (dabali) et « travail » (baara), sont normalement utilisés pour faire allusion, de manière euphémistique, aux « pratiques » de la magie. Il en résulte ainsi que la guérisseuse n'utiliserait pas la divination. Or, dans une seconde phase, suite à une demande plus précise, j'obtiens la réponse : « Oui, quand un malade arrive, je l'examine d'abord en consultant les cauris que j'étale sur le sol. ». Dans l'intention louable de m'aider comprendre, $\mathrm{AC}$ prend alors l'initiative et demande à la jinètigi de lancer les coquillages à titre d'exemple. Sika s'exécute et explique. Selon la traduction de $\mathrm{AC}$, les cauris indiquent que " dans une semaine », deux personnes accompagneront un malade souffrant de mal de tête pour consulter la jinètigi. Prenant la traduction « dans une semaine » au sens littéral, je mets la pression sur AC, déterminée par la volonté de préciser une fois pour toute à mon interprète la différence entre 
interprétation et traduction, mieux, la limite que je souhaitais alors établir entre les deux termes-concepts, et ceci jusqu'à ce qu'il fût contraint de confesser : « Non, la semaine ... elle n'a pas précisé ». Ainsi, après une vérification ultérieure avec ST, où la guérisseuse explique qu'évidemment, il s'agissait seulement d'une démonstration à finalité didactique, bien différente de ce qui se déroule durant la cérémonie, $\mathrm{AC}$ se justifia par « Je vais tout dire, eh ! Moi je suis clair envers toi aussi ! »

Toujours dans cette optique, un dernier passage de l'entretien me semble utile à analyser (Tab. 4). C'est grâce à un énième malentendu, en effet, que j'obtiens une explication causale, assez intéressante, du surmenage.

ST parle de la délusion éprouvée par qui, après avoir beaucoup étudié à l'école des Tubabu (des Blancs), n'obtient (litt.: « trouve », soro) rien. A vrai dire, elle s'étonne même («Hé !») que, seuls, nous ne parvenions pas à comprendre le potentiel psychopathogène des délusions de ce genre :

\section{Tableau 4}

AC. Elle dit qu'il y a deux sortes de fous : l'autre là c'est le marabout, et l'autre là c'est le jine.

MLC. Et pour ce qui est de l'étude ? Elle a dit d'abord qu'on peut étudier trop, et donc on peut avoir le surmenage lié à l'étude, mais est-ce que ça se passe pour l'école ou pour l'étude du Coran?

AC. Dis, maintenant, faire des études à faire entrer la folie en l'homme, l'étude scolaire [lecolikalan] est plus probable [teli, rapide] dans cela ou plutôt c'est l'étude coranique [morikalan] qui est le plus rapide dans cela?
AC. Ko sisan, ka kàlan ke ka fa kari mogo la, yali ecolikalan de ka teli n'o ye wa morikalan de ka telin'o ye?
ST. Hum, c'est le morikalan qui est le plus rapide.
ST. Hum, morikalan ka teli n'o ye.

MLC. Il n'y a pas de danger pour l'école...

AC. Non, il n'y a pas de danger pour l'école, mais pour les marabout c'est très dangereux [la jinetigi rit]. Donc tu as compris ça ? [en riant].

MLC. Un peu [en riant].

MLC. Mais le problème quand on n'a pas d'emploi ? Elle avait dit que c'est dangereux le fait de trop étudier si on n'a pas d'emploi, mais pourquoi ça ? 


\begin{abstract}
AC. Bon, comme maintenant, une personne qui a fait assez de tubabukalan [les études des Tubabu, les Blancs], eh, si elle n'y gagne rien, son $j a$ peut-il se lever pour devenir de la folie ? Comment cela se fait-t-il ?
\end{abstract}

\begin{tabular}{|c|c|}
\hline $\begin{array}{l}\text { ST. Hé ! Quand tu ne trouves } \\
\text { rien dans le tubabukalan, si } \\
\text { cela ne n'est pas maîtrisé par } \\
\text { ton cœur [dusu.kun, cœur.tête], } \\
\text { cela devient la folie. Comme } \\
\text { quelqu'un gagne beaucoup } \\
\text { d'argent, s'il va en faillite, toi- } \\
\text { même tu sais qu'il s'agit de ta } \\
\text { grande fortune, si elle se gâte } \\
\text { pour toi, quand tu vois qu'il } \\
\text { n'existe aucune possibilité de } \\
\text { la récupérer, bon, cela conduit } \\
\text { à parler de hakili nyagami, il } \\
\text { va donc confondre [nyagami, } \\
\text { mélanger] ton esprit [hakili]. }\end{array}$ & 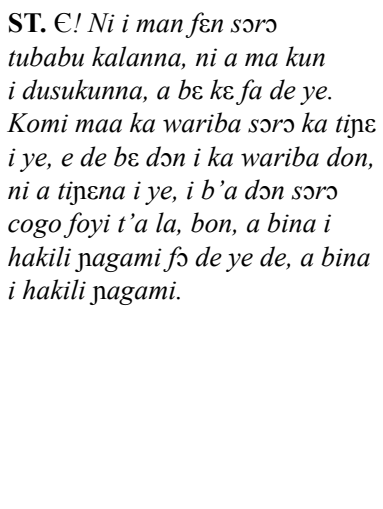 \\
\hline
\end{tabular}

ST. Hé ! Quand tu ne trouves rien dans le tubabukalan, si quelqu'un gagne beaucoup d'argent, s'il va en faillite, toin'existe aucune possibilité de la récupérer, bon, cela conduit mélanger] ton esprit [hakili].
AC. Bon, komi sisan, u koni mogo ye tubabukalan ke kosebs, hum, ni ma fen soro a la, a ja be wuli ka ke fa ye, o be se ka $k \varepsilon$ cogo jumena?
AC. Ce qu'elle dit maintenant, elle dit qu'il y a deux façons : par exemple quand quelqu'un qui a instruit beaucoup, qui n'a pas eu d'emploi, directement il faut avoir un emploi, s'il n'a pas eu, directement ça rend fou. Mais il y a deux cas : par exemple quelqu'un qui a beaucoup d'argent, si l'argent est perdu, ça aussi ça rend fou.

Le cas de l'homme qui a investi dans ses études sans en tirer les avantages espérés « un emploi », dit AC - est comparé à la situation de celui qui aurait perdu une bonne quantité d'argent sans espoir de récupération. Si le cœur (dusukun) ne peut faire front ou faire face (kun, qui comme substantif signifie tête) à la situation, le hakili (l'esprit) devient confus (nagami : se mélange), et « ça rend fou » (traduit AC). Se fait jour ici une explication de type psychologique ou psycho-dynamique : la perte des biens, le désir non satisfait, l'échec d'un projet d'importance vitale sont des causes de folie que d'autres guérisseurs de kunkolobana mentionnent également (Ciminelli, 1998a). Les circonstances citées en exemple présentent leur spécificité et coïncident avec celles que rappellent deux guérisseurs interviewés par Bastien (1988:43, 70-71, 166-167) à propos du dusukasi (cœur qui pleure). Dusukasi, traduit par « angoisse » ou « anxiété » en termes psychiatriques, est considéré comme un symptôme de dépression (Koumaré, Coudray, Kounaté, 1984 ; Ben Baba, 1984 : 76). Il convient alors de partager pleinement l'importante observation formulée par Maurice Dorès à propos du terme wolof analogue de naxar ( « chagrin ») : le fait que de telles causes, souvent évoquées par les guérisseurs, ne soient pas spectaculaires comme les causes « magiques » a probablement déterminé leur omission dans les recherches, ou dans l'analyse des données (Dorès, 1981 : 45). 
Et la prolifération de syndromes « exotiques » ou de culture-bound syndromes, même inexistants en tant que maladies psychiatriques ${ }^{29}$, va certainement en appui à cette thèse.

Nous sommes ainsi à la fin du dialogue : à cet instant se manifeste également la fatigue, voire l'épuisement (Tab. 5).

Je n'arrive pas à trouver les mots justes pour parler de la dépression : je ne me rappelle pas non plus du terme français « désespoir », tandis que la locution « le moral gâté », qui traduit souvent l'état d'âme du déprimé (Ciminelli, 1998a : 222-223), n'est pas saisi par AC et n'est pas traduit, comme j'espérais, par diinc tincn. En outre, la traduction en bamanankan du mot « espoir », à savoir jigi - qui signifie également « personne sur laquelle on compte »- suscite une équivoque ultérieure entre AC et la guérisseuse-même, qui lui donne la description de l'« idiot» (nalonma) : celui qui «ne pense à rien, ne réfléchit pas, n'a pas d'espoir, n'a pas de possibilité ». Finalement, l'entretien se termine par un quiproquo comique : je crois que le mot français « idiot», que AC me fournit en le prononçant " idjot » (transcrit "ijo " dans le texte infra) est précisément le mot bamanankan auquel je m'attendais comme traduction de « déprimé ». Certes, comme le disait Bergson (1900: 99), « cette équivoque n'est pas comique par elle-même ; elle ne l'est que parce qu'elle manifeste la coïncidence des deux séries indépendantes » : à savoir, dans notre cas, les deux discours qui se déroulent, indépendamment l'un de l'autre, en français et en bamanankan. Heureusement, à ce point-là la cassette se termine et on rentre à la maison.

Est-il possible de formuler des remarques conclusives par rapport à ce récit de terrain ?

Comme je l'ai indiqué au début de ce texte, le lieu du travail anthropologique se situe probablement dans l'écart entre la compréhension et son contraire, l'incompréhension. Cet écart prend des formes variées, qui évoluent au cours de la recherche; le malentendu est l'une des plus importantes à analyser. Ce travail, étant d'ordre récursif en tant qu'il implique un constant et double « retour » tant sur l'objet que sur le sujet de connaissance, n'a donc pas une véritable « conclusion ».

29 Certains « syndromes liés à la culture » ou culture-bound syndromes (v. supra, note 18) se sont révélés être des « inventions » occidentales : parmi ceux-ci, le cas le plus fameux est celui du wiitiko ou windigo des Natives du nord-est américain, un prétendu syndrome de cannibalisme compulsif qui n'est en réalité qu'une légende algonquine (Ciminelli, 1997b, 1998a, 1998b). 
Tableau 5

\begin{tabular}{|c|c|c|}
\hline & & $\begin{array}{l}\text { MLC. Toujours [au sujet du] } \\
\text { jawuli. Elle a dit «dusukun » } \\
\text { pour le premier cas, celui } \\
\text { qui concerne le type qui } \\
\text { a beaucoup étudié et qui } \\
\text { n'a pas trouvé d'emploi. } \\
\text { Qu'est-ce que dusukun? }\end{array}$ \\
\hline $\begin{array}{l}\text { AC. Qu'est-ce que } \\
\text { c'est le dusukun? }\end{array}$ & AC. E, dusukun ye mun ye? & \\
\hline \multirow{7}{*}{$\begin{array}{l}\text { ST. Dusukun? Il se trouve } \\
\text { au niveau de la poitrine de la } \\
\text { personne [elle se touche la } \\
\text { poitrine au niveau du cœur]. }\end{array}$} & $\begin{array}{l}\text { ST. Dusukun? Є, a be maa } \\
\text { yere dusukun de la yan. }\end{array}$ & \\
\hline & & AC. Dusukun c'est le cœur. \\
\hline & & $\begin{array}{l}\text { MLC. Si l'on doit traduire } \\
\text { désespération [sic] - ça existe } \\
\text { en français j'espère - bon, } \\
\text { quand on est désespéré, on } \\
\text { n'a pas d'espoir, qu'est-ce } \\
\text { qu'on peut dire en bambara? }\end{array}$ \\
\hline & & $\begin{array}{l}\text { AC. On n'a pas d'espoir en } \\
\text { bambara, il n'a rien, quoi ? } \\
\text { C'est-à-dire le savoir, quoi ? }\end{array}$ \\
\hline & & $\begin{array}{l}\text { MLC. Non, quand une } \\
\text { personne est déprimée, } \\
\text { qui n'a pas d'espoir ... }\end{array}$ \\
\hline & & $\begin{array}{l}\text { AC. Qui n'a pas d'espoir } \\
\text { dans la vie, quoi ? }\end{array}$ \\
\hline & . & $\begin{array}{l}\text { MLC. Voilà, qui se sent } \\
\text { vraiment ... avec le moral } \\
\text { gâté et tout ça ... }\end{array}$ \\
\hline $\begin{array}{l}\text { AC. Bon, dis maintenant, } \\
\text { une personne qui n'est } \\
\text { dans rien de vivant comme } \\
\text { espoir, qui n'a personne } \\
\text { comme support d'espérance, } \\
\text { comment est cette personne? } \\
\text { Que dire de son cas? }\end{array}$ & $\begin{array}{l}\text { AC. Bon, ko sisan, məgə } \\
\text { min ni... c'est-à-dire, a ti } \\
\text { ni fen werela, a jigila foyi, } \\
\text { c'est-à-dire jigila mogə t'a } \\
\text { bolo : fo a be a [...] mogə be } \\
\text { di, o ka ko fana be cogodi? }\end{array}$ & \\
\hline $\begin{array}{l}\text { ST. Une personne qui est } \\
\text { dans ce cas, qui ne pense à } \\
\text { rien dans la case du monde? }\end{array}$ & $\begin{array}{l}\text { ST. } \epsilon, \text { mogo min be } \\
\text { ro e te miiri foyi foyi } \\
\text { la dine so k๖n॰? }\end{array}$ & \\
\hline $\begin{array}{l}\text { AC. C'est-à dire, tu n'as } \\
\text { rien, tu n'as aucun pouvoir. }\end{array}$ & $\begin{array}{l}\text { AC. C'est-à-dire fen } t{ }^{\prime} i \\
\text { bolo quoi, se t'i ye, quoi. }\end{array}$ & \\
\hline
\end{tabular}




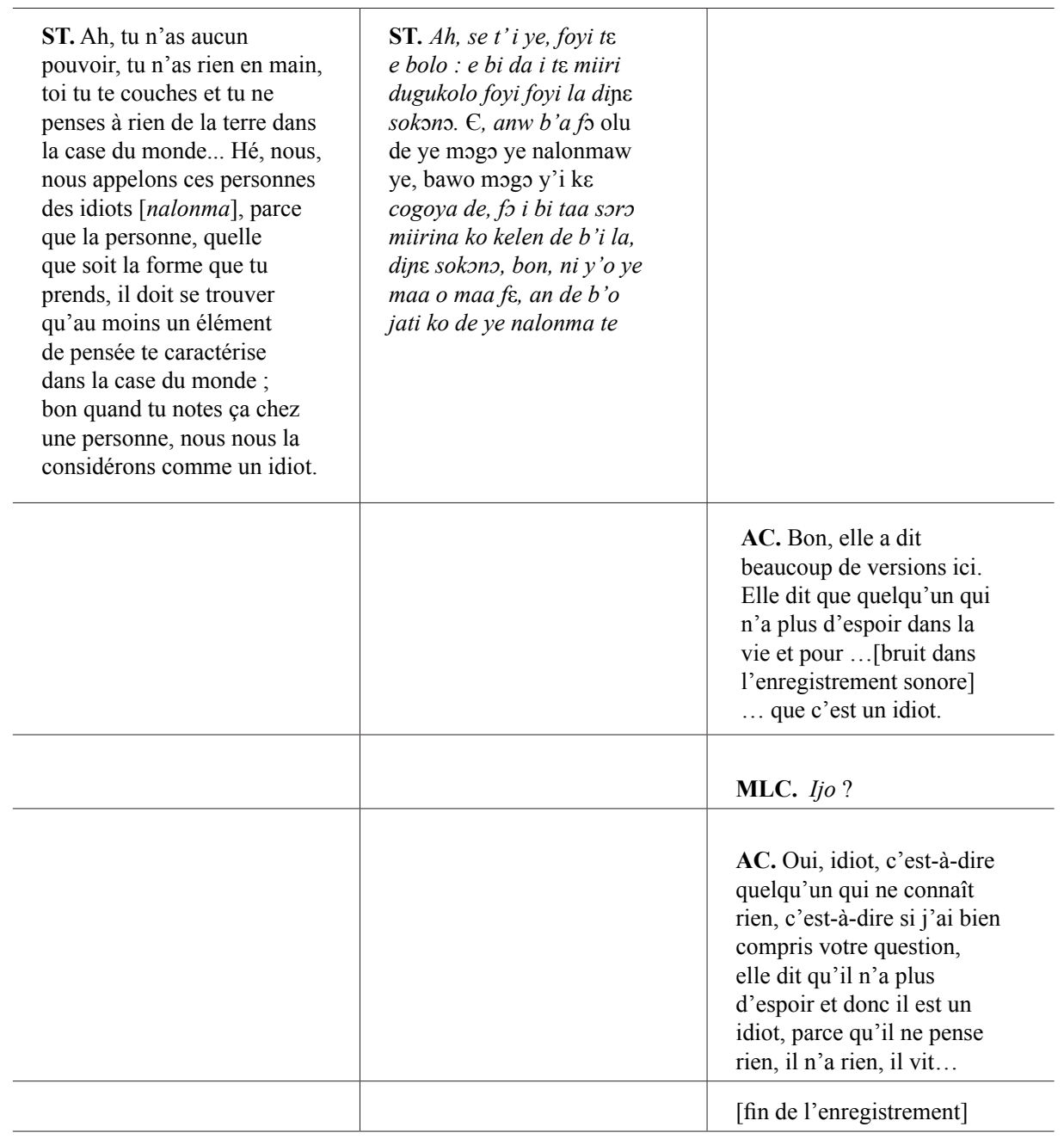

Dans cette étude, j'ai essayé de mettre en évidence, à travers quelques exemples extraits de mon parcours de recherche, les pièges de la traduction « culturelle », dans laquelle les malentendus se produisent (au niveau soit de la traduction « intermodale », soit de la traduction proprement dite ou linguistique) abstraction faite de la composante psychologique, qui peut certainement influer sur l'interprétation ou sur la mésinterprétation des faits, gestes, discours, expressions. Il m'a semblé important de rappeler et d'insister sur le fait que chaque traduction est orientée par la culture d'appartenance du sujet qui traduit ; puis de mettre en évidence comment le rapport asymétrique, que pose en soi chaque acte de traduction, entre sujet et objet de la traduction, peut se superposer à, et agir en synergie avec, cette asymétrie de pouvoir existant entre cultures et langues dominantes et subalternes, laquelle fut mise en évidence pour la première fois par Talal Asad (1986). Suivant une indication précoce de Roger Keesing (1984), j'ai voulu insister sur le fait que l'un des malentendus 
culturels les plus fréquents se fonde sur l'interprétation « ontique » de certains façons de dire («ways of talking ») qui sont en réalité d'ordre métaphorique, représentent plus précisément des métaphores de type « cognitif», en référence à l'étude développée par George Lakoff et Mark Johnson. Une autre notion, celle d' " opposition hiérarchique » de Louis Dumont, m'est apparue comme un instrument utile pour comprendre les " significations spécifiques » de certains termes-concepts, culturellement centraux mais pour autant assez « vagues » comme, dans le cas spécifique, le terme-concept de « santé ». Finalement, réexaminer la transcription d'un entretien de terrain a fait émerger non seulement ces « manipulations » dans la traduction par l'interprète, que j'avais toutefois devinées sur le terrain, mais plutôt leur motivation et raisons d'être, illuminant, par exemple, ses précautions linguistiques d'ordre pragmatique, qui m'avaient alors totalement échappé. Ainsi, avec le temps, les « déformations », les « inexactitudes » ou les « erreurs » de ces traductions se sont révélées autrement « informatives » que les traductions dont j'étais satisfaite.

Je voudrais donc terminer cet écrit en soulignant, avec Johannes Fabian, l'importance de reporter dans nos ethnographies non seulement ce que nous, les anthropologues, comprenons, mais aussi ce que l'on ne comprend pas, « even on the level of interpreting grammatical and lexical meaning » (Fabian, $1995: 43$ ). En fait, si la traduction, comme le dit le même auteur, "requires historical background knowledge " (ibid.), cette connaissance historique devrait aussi inclure une réflexion personnelle sur sa propre expérience concernant le malentendu « ethnographique » ou « culturel».

\section{Références citées}

AdAm, Jean-Michel, 1990. «Aspects du récit en anthropologie », in Jean-Michel Adam, Marie-Jeanne Borel, Claude Calame et Mondher Kilani (éds), Le discours anthropologique. Description, narration, savoir, pp. 251-282. Paris : Méridiens Klincksieck.

Affergan, Francis, 1987. Exotisme et altérité. Essai sur les fondements d'une critique de l'anthropologie. Paris : PUF.

Agamben Giorgio, 1995. Homo sacer. Il potere sovrano e la nuda vita. Turin : Einaudi.

AsAD, Talal, 1986. " The concept of cultural translation in British social anthropology », in James Clifford et George E. Marcus éds), Writing culture : The politics and poetics of ethnography, pp. 141-64. Berkeley : University of California Press.

Austin, John L., 1975. How to do things with words. Oxford : Oxford University Press.

BaILleul, Charles et al., 2011-2014. Bamadaba : Dictionnaire électronique bambara-français, avec un index français-bambara. <http://cormand.huma-num.fr/bamadaba.html> (consulté en septembre 2015).

Bastien, Christine, 1988. Folies, mythes et magies d'Afrique noire. Propos des guérisseurs du Mali. Paris : L'Harmattan.

Ben BABA, Arwata, 1984. Contribution à l'étude des états dépressifs au Mali. Thèse en Médecine. Bamako : Ecole Nationale de Médecine et de Pharmacie du Mali.

Benjamin, Walter, 1969. Illuminations. New York : Schocken.

Bergson, Henri, 1900. Le rire : essai sur la signification du comique. Paris : PUF.

Bonnet, Doris, 1986. Représentations culturelles du paludisme chez les Moose du Burkina, document Orstom. Ouagadougou : Orstom. 
Borel, Marie-Jeanne, 1990. « Le discours descriptif, le savoir et les signes », in Jean-Michel Adam, MarieJeanne Borel, Claude Calame et Mondher Kilani (éds), Le discours anthropologique. Description, narration, savoir, pp. 11-69. Paris : Méridiens Klincksieck.

Borutti, Silvana, 1991. Teoria ed interpretazione. Per una epistemologia delle scienze umane. Milan : Guerini.

Bourdieu, Pierre, 1980. Le sens pratique. Paris : Editions de Minuit.

Bouveresse, Jacques, 1981. «Wittgenstein et la philosophie du langage », in Guttorm Floistad (éd.), Contemporary philosophy. A new survey, 1, pp. 83-112. La Haye-Boston-Londres : Martinus Nijhoff.

CAnguilhem, Georges, 1966 [1947]. Le normal et le pathologique. Paris : PUF.

CANUT, Cécile, 2008. Le spectre identitaire. Entre langue et pouvoir au Mali. Limoges : Lambert-Lucas.

Ciminelli, Maria Luisa, 1992. «Per una ricerca etnopsichiatrica a Bamako (Mali). Surmenage mental : culture-bound syndrome o traduzione ? Un problema di dinamica culturale », L'Uomo, 5, pp. 87-104.

-, 1996. « Tubabu in Mali. Statuto dell'osservatore e controtransfert culturale nel fieldwork », Africa, 51, pp. 587-600.

-, 1997a. "Kènè e le sue metafore. Note sul sistema salute/malattia in ambito bamanan », in Roberto Beneduce (éd.), Saperi, linguaggi e tecniche nei sistemi di cura tradizionali, pp. 123-155. Turin : L'Harmattan Italia.

-, 1997b. « "Culture-bound syndrome": un concetto vago e di dubbia utilità », AM, 3-4, pp. 247-280.

-, 1998a. Follia del sapere e saperi della follia : Percorsi etnopsichiatrici tra i Bamanan del Mali. Milan : Franco Angeli.

-, 1998b. «La decostruzione del concetto di “culture-bound syndrome” », in Vittorio Lanternari et Maria Luisa Ciminelli (éds), Medicina, magia, religione, valori. Vol. II : Dall'antropologia all'etnopsichiatria, pp. 85-107. Naples : Liguori Editore.

-, 2000. « Coltura e cultura : metafore relative a vita, salute, malattia tra i Bamanan del Mali », in G. Volpato (éd.), Agricoltura, musei, trasmissione dei saperi, pp. 299-308. Vérone : Accademia di Agricoltura Scienze e Lettere.

-, 2004. «Nama (nyama) as cause of evil and illness : Analysis of a manaconcept in the Bamana context », Quaderni di Semantica, 25 (1), pp. 9-23.

-, 2005. «Politiche della salute e tradizioni "orientate" di termini di malattia. Alcuni esempi relativi alle lingue mandinghe (Africa Occidentale) », La Ricerca Folklorica, 50, pp. 23-34.

-, 2008a. D'incanto in incanto : Storia del consumo di arte primitiva in Occidente. Boulogne : Clueb.

-, 2008b. « Salvaguardia del patrimonio culturale immateriale e possibili effetti collaterali : etnomimesi ed etnogenesi », in Lauso Zagato (éd.), Le identità culturali nei recenti strumenti Unesco : un approccio nuovo alla costruzione della pace?, pp. 99-122. Padoue : Cedam.

-, 2009. « Djenné, Timbuctu, il Pays Dogon : protezione del patrimonio culturale ed eterogenesi dei fini », Africa e Mediterraneo, 65-66, pp. 36-39.

-, 2011. « Ritorno a Mashpee : alcuni problemi antropologici trasversali nei nuovi strumenti internazionali a difesa delle culture locali », in Luisa Faldini et Eliana Pili (éds), Saperi antropologici, media e società civile nell'Italia contemporanea, pp. 321-330. Rome : Cisu.

-, 2013. « La Biennale, l'antropologia, gli indigeni », AM-Antropologia Museale, 11 (32/33), pp. 63-68.

-, 2016. « Tempi diseguali : Coevità e contemporaneità in arte », Rivista di estetica, 61 (1), pp. 17-33.

DiAkité, Djigui, 1989. Essai sur les traditions sanitaires et médicinales Bambara du Bélédougou. Thèse en Médecine. Bamako : Ecole Nationale de Médecine et de Pharmacie du Mali. 
DiaourÉ, Ramata, 1992. «Les dénominations des malaises et des maladies en bamanan », in André Marcel et al. D’ans, Langues et métiers modernes ou modernisés au Mali. Santé et travail du fer, pp. 145-149. Paris : Institut d'études créoles et francophones-Didier.

DiARra, Tiéman, 1977. La réticence des parents à la scolarisation en milieu traditionnel bambara du Bélédougou. Mémoire de Psychopédagogie. Bamako : Ecole Nationale Supérieure en PsychoPédagogie.

Dorès, Maurice, 1981. La femme village. Maladies mentales et guérisseurs en Afrique Noire. Paris : L'Harmattan.

Dumont, Louis, 1979. Homo hierarchicus. Le système des castes et ses implications. Paris : Gallimard.

-, 1983. Essais sur l'individualisme : une perspective anthropologique sur l'idéologie moderne. Paris : Seuil.

-, 1988 [1971]. Introduction à deux théories d'anthropologie sociale. Groupes de filiation et alliance de mariage. Paris : Ehess.

Eco, Umberto, 2003. Dire quasi la stessa cosa : Esperienze di traduzione. Milan : Bompiani.

FABIAN, Johannes, 1995. " Ethnographic misunderstanding and the perils of context ", American Anthropologist, 97 (1), pp. 41-50.

FAssin, Didier, 1992. Pouvoir et maladie en Afrique. Anthropologie sociale dans la banlieue de Dakar. Paris : PUF.

Gellner, Ernest, 1970. « Concepts and society », in Bryan R. Wilson (ed.), Rationality, pp. 18-49. Oxford : Basil Blackwell.

GibBal, Jean-Marie, 1982. Tambours d'eau. Journal et enquête sur un culte de possession au Mali occidental. Paris : Le Sycomore.

Goodman, Nelson, 1978. Ways of worldmaking. Indianapolis : Hackett.

Graburn, Nelson H., 1976. Ethnic and tourist arts. Cultural expression from the Fourth World. Berkeley : University of California Press.

Grice, Paul, 1989. Studies in the way of words. Cambridge: Harvard University Press.

Hanks, William F. et Carlo Severi, 2014. « Translating worlds. The epistemological space of translation », Hau : Journal of Ethnographic Theory, 4 (2), pp. 1-16.

HANKs, William F., 2014. « The space of translation », Hau : Journal of Ethnographic Theory, 4 (2), pp. 1739.

IVIR, Vladimir, 1977. « Lexical gaps : a contrastive view », Studia Romanica et Anglica Zagrabiensia, 43, pp. 167-176.

JAKOBSON, Roman, 1959. « Linguistic aspects of translation », in Reuben A. Brower (éd.), On translation, pp. 232-238. Cambridge : Harvard University Press.

KeEsing, Roger M., 1985. « Conventional metaphors and anthropological metaphysics : the problematic of cultural translations », Journal of Anthropological Research, 41 (2), pp. 201-218.

-, 1987, « Anthropology as interpretive quest », Current Anthropology, 28 (2), pp. 161-169.

KiLANI, Mondher, 1990a. " Les anthropologues et leur savoir : du terrain au texte », in Jean-Michel Adam, Marie-Jeanne Borel, Claude Calame et Mondher Kilani (éds), Le discours anthropologique. Description, narration, savoir, pp. 71-109. Paris : Méridiens Klincksieck.

-, 1990b. « Que de hau! le débat autour de l'Essai sur le don et la construction de l'objet en anthropologie », in Jean-Michel Adam, Marie-Jeanne Borel, Claude Calame, Mondher Kilani (éds), Le discours anthropologique. Description, narration, savoir, pp. 135-168. Paris : Méridiens Klincksieck.

-, 1994. L'invention de l'autre. Essais sur le discours anthropologique. Paris : Payot. 
Koné, Zakaria, 1978. Etude psychopathologique des céphalées chez les scolarisés du second cycle fondamental. Mémoire de Psychopédagogie. Bamako : Ecole Nationale Supérieure en PsychoPédagogie.

Kosкo, Bart, 1993. Fuzzy thinking : the new science of fuzzy logic. New York : Hyperion.

Koumaré, Baba, Jean-Pierre Coudray, 1986. « Jinè Bana : un concept opératoire ? », Psychologie Médicale, 18 (12), pp. 1811-1813.

Koumaré, Baba, Jean-Pierre Coudray, Fernand Kanouté, 1984. « Les états dépressifs 'insolites’ au Mali : vers une unité nosologique », Psychiatrie Francophone, 1, pp. 22-27.

LAKoff, George, 1987. Women, fire and other dangerous things. Chicago : Chicago University Press.

-, 1993. «The contemporary theory of metaphor », in Andrew Ortony (éd.), Metaphor and thought, pp. 202251. Cambridge : Cambridge University Press.

LAKOFF, George, et Mark JoHnson, 1980a. « The metaphorical structure of the human conceptual system », Cognitive Science, 4, pp. 195-208.

-, 1980b. Metaphors we live by. Chicago : Chicago University Press.

LePENIES, Wolf, 1993. « Traducibilità delle culture », Prometeo, 11 (44), pp. 6-17.

Malinowski, Bronislaw, 1935. Coral gardens and their magic. Volume two : The language of magic and gardening. Londres : George Allen and Unwin Ltd.

Mauss, Marcel, 1950. " Les techniques du corps », Sociologie et Anthropologie, pp. 365-386. Paris : PUF. [Journal de Psychologie, 1936].

Miceli, Silvana, 1982. In nome del segno. Introduzione alla semiotica della cultura. Palerme : Sellerio.

-, 1990. Orizzonti incrociati. Il problema epistemologico in antropologia. Palerme : Sellerio.

PiAsere, Leonardo, 2002. L'etnografo imperfetto. Rome-Bari : Laterza.

Polia, Mario, 1998. « Le sindromi culturali da perdita della sombra nel curanderismo andino del Perù settentrionale », in Vittorio Lanternari et Maria Luisa Ciminelli (éds), Medicina, magia, religione, valori. Volume II : Dall'antropologia all'etnopsichiatria, pp. 301-318. Naples : Liguori Editore.

Prince, Raymond, 1960. " 'The brain-fag' syndrome in Nigerian students », Journal of Mental Sciences, 106, pp. 559-570.

Roger, Miriam, 1993. " Sumaya : le palu et le reste (région de Sikasso) », in Joseph Brunet Jailly (éd.), Se soigner au Mali. Une contribution des sciences sociales, pp. 83-126. Paris : Karthala.

SBISÀ, Marina (éd.), 1993. Gli atti linguistici. Aspetti e problemi di filosofia del linguaggio. Milan : Feltrinelli.

SEARle, John R., 1969. Speech acts : An essay in the philosophy of language. Cambridge : Cambridge University Press.

Sindzingre, Nicole, 1984. " La nécessité du sens : l'explication de l'infortune chez les Senufo », in Marc Augé et Claudine Herzlich (éds), Le sens du mal. Anthropologie, histoire, sociologie de la maladie, pp. 93-122. Paris/Montreux : Editions Archives contemporaines.

Stasch, Rupert. 2014. « Powers of incomprehension. Linguistic otherness, translators, and political structure in New Guinea tourism encounters », Hau : Journal of Ethnographic Theory, 4 (2), pp. 73-94.

Wittgenstein, Ludwig, 1953. Philosophische Untersuchungen. Oxford : Basil Blackwell.

-, 1977. Remarks on colour. Oxford : Basil Blackwell. 